\title{
Laser speckle flow graph-assisted sympathetic gangliectomy for treatment of facial blushing: Can technology aid psychology?
}

\author{
Eugenio Pompeo, MD, FETCS
}

From the Department of Thoracic Surgery, Policlinico Tor Vergata University, Rome, Italy

Disclosures: Author has nothing to disclose with regard to commercial support.

Received for publication Jan 28, 2018; accepted for publication Jan 31, 2018; available ahead of print March 7, 2018

Address for reprints: Eugenio Pompeo, MD, FETCS, Department of Thoracic Surgery, Policlinico Tor Vergata

University, Via le Oxford 81, 00133 Rome, Italy (E-mail: pompeo@med.uniroma2.it).

J Thorac Cardiovasc Surg 2018;156:1332-3

$0022-5223 / \$ 36.00$

Copyright (c) 2018 by The American Association for Thoracic Surgery

https://doi.org/10.1016/j.jtcvs.2018.01.077

Facial blushing, a sudden redness of the face triggered by emotional or social stress, is regarded as the hallmark of embarrassment and is often present in social anxiety disorders. It was defined in 1872 by Charles Darwin ${ }^{1}$ as the most peculiar of all human expressions of emotions and strictly related to what other people think of us. Although in most circumstances it constitutes an adequate social signal, it may sometimes become an invalidating condition that can significantly impair social interaction and quality of life. ${ }^{2}$ Thoracoscopic sympathectomy for treatment of facial blushing was first reported in the 1980 s by Wittmoser ${ }^{3}$ but has gained in popularity in recent years.

In this issue of the Journal, Yamamoto and Okada, ${ }^{4}$ on the basis of an impressive previous experience of 12,000 sympathectomies, present to the readers a study dealing with an interesting novel high-tech method for intraoperative identification of the neural pathway underlying recurrent facial blushing after previous sympathectomy and eventually guiding redo thoracoscopic ganglionectomy. The method of Yamamoto and Okada ${ }^{4}$ is based on intraoperative monitoring of the vasomotor sympathetic nerve function by a laser speckle flow graph (LSFG) device, which can noninvasively evaluate changes in skin blood perfusion according to the "laser speckle phenomenon." Yamamoto and Okada ${ }^{4}$ underline that the target neural circuit relating to facial blushing can be identified by LSFG as the sympathetic nerves and ganglions where transient decreases in facial skin blood perfusion are observed after their selective electrostimulation. With this method, satisfactory results were achieved in 8 patients with postsympathectomy unilateral (6 patients) or bilateral (2 patients) recurrent symptoms. Overall, all patients declared themselves to be very satisfied ( 6 patients) or satisfied ( 2 patients) with the reoperation.

Given that interpreting LSFG images is probably not such an easy game as one might optimistically believe, Yamamoto and $\mathrm{Okada}^{4}$ are to be commended for having

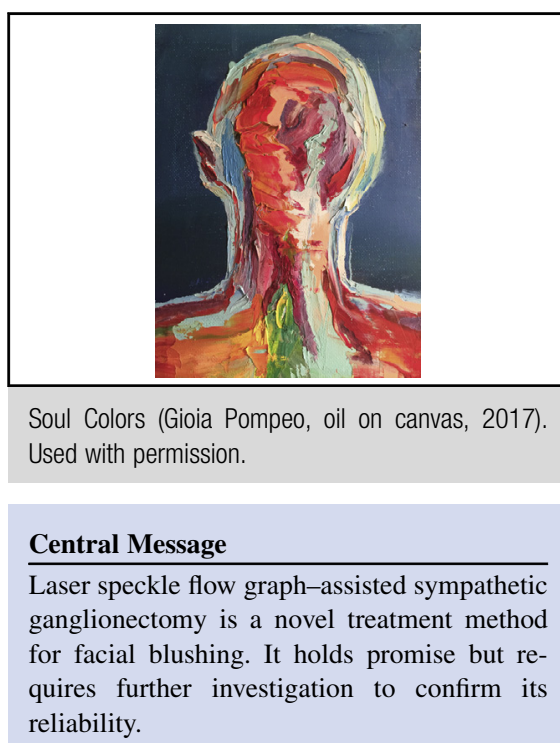

See Article page 1326. provided us an excellent study. On the other hand, a few notes of caution seem mandatory. In fact, concern may be raised regarding the reproducibility of this method, which should be assessed in the near future within well designed unicenter and multicenter controlled studies. In addition, though LSFG monitoring can be considered a sort of realtime objective prediction of the ganglionectomy effect, the final outcome measure in this study was based on a patient's perceived subjective evaluation of improvement, whereas a more reliable, objective, quantitative outcome measure would have been preferable in this regard.

Nonsurgical treatment, including selective serotonin reuptake inhibitors, $\beta$-receptor blockers, and particularly psychologic treatment, must be considered the first line treatment for facial blushing, and patients must be precisely informed of the pros and cons of surgical ganglionectomy, including the risk of development of severe adverse effects. Nonetheless, LSFG-assisted ganglionectomy may be considered as a promising novel tool that could radically change the mode of treatment of patients with some of the more complex sympathetic trunk-related disorders, including patients with unilateral recurrence of facial blushing symptoms, or Harlequin syndrome, ${ }^{2}$ which may sometimes be perceived as an even more severely invalidating condition than the initial bilateral facial blushing itself. 
In conclusion, although these preliminary results must be taken with caution and further careful investigation is warranted, Yamamoto and Okada ${ }^{4}$ have presented an exquisite and as yet quite rare example of how surgical technology can sometimes modify physiology and aid psychology.

I thank Ms Gioia Pompeo for permission to use a personal painting image.

\section{References}

1. Darwin C. The expression of the emotions in man and animals. In: Porter DM, Graham PW, eds. The Portable Darwin. New York: Penguin Books; 1993:364-93.

2. Kristian S, Christer D. Facial blushing patient selection and long-term results. Thorac Surg Clin. 2016;26:459-63.

3. Wittmoser R. Treatment of sweating and blushing by endoscopic surgery. Acta Neurochir. 1985;74:153-4.

4. Yamamoto H, Okada M. Sympathetic ganglionectomy for facial blushing using application of laser speckle flow graph. J Thorac Cardiovasc Surg. 2018;156: 1326-31. 